\title{
Donald Arthur Glaser (1926-2013)
}

Physicist and biotechnologist who invented the bubble chamber.

$\mathrm{D}$ onald Arthur Glaser was a great physicist and one of the first biotechnology entrepreneurs. He was also the least arrogant, most delightfully funny person you could hope to meet. He was aged just 25 when he designed and built the first bubble chamber, a device that allowed physicists to visualize with unprecedented precision the trajectory of elementary particles, and so measure their properties.

Glaser, who died on 28 February, was born in 1926, in Cleveland, Ohio. He received a bachelor's degree in physics and mathematics in 1946 from Cleveland's Case School of Applied Science (now Case Western Reserve University). As a child, he loved the arts especially music - and while at Case he played the viola in the Cleveland Philharmonic Orchestra.

After his degree, Glaser started a PhD in physics at the California Institute of Technology (Caltech) in Pasadena. His interest in particle physics led him to work with Nobel laureate Carl Anderson, studying cosmic rays. To do this, he used a cloud chamber, an early particle detector that was essentially a sealed chamber filled with vapour. During these years, Glaser learned to design and build the equipment he needed for his experiments, a skill that served him well when he became an assistant professor at the University of Michigan in Ann Arbor in 1949.

It was at Michigan that Glaser built the first bubble chamber, an improvement on the cloud chamber. Whereas the cloud chamber was filled with vapour, the bubble chamber is filled with liquid. A piston produces a sudden decrease in pressure, forcing the liquid into a superheated state. Charged particles create an ionization track around which the liquid vaporizes, forming microscopic bubbles; the density of bubbles is proportional to the particle's energy loss. The device had a profound effect on particle physics, enabling the use of ever larger accelerators.

Glaser possessed a charming mix of curiosity and innocence. For a physicist considering how to visualize elementary particles, a natural step would have been to consult the relevant chapter in Enrico Fermi's 1937 book Thermodynamics, the reference text for particle physics. But instead Glaser worked through his own calculations from scratch. Had he read Fermi's book, he would have seen an equation that

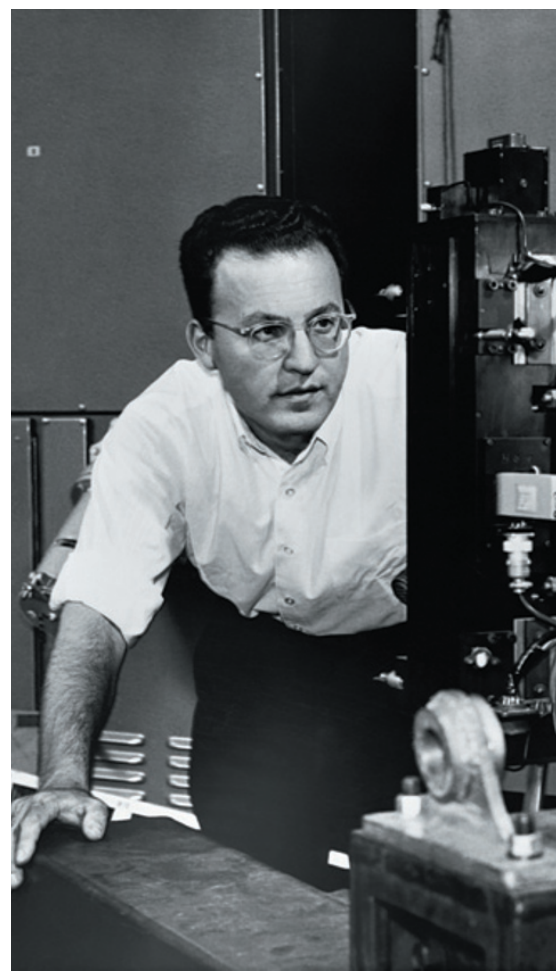

implied that the bubble chamber could not work. Fermi had an iron-clad reputation for not making mistakes, but he had made one here, leaving the door open for Glaser's great invention.

In 1960, at the age of just 34, Glaser received the Nobel Prize in Physics for his work on the bubble chamber. Glaser's supervisor Anderson was the student of Robert Millikan, another physics Nobel prizewinner from Caltech. According to Glaser, his students used to look at one another and wonder who would be the fourth in this distinguished line. In 1993, the Nobel Prize in Chemistry did go to someone with whom Glaser had worked. That person was biochemist Kary Mullis, who received the prize for inventing the polymerase chain reaction (PCR), which can amplify one or a few pieces of DNA into thousands or millions of copies. Yet the connection between Glaser and Mullis had nothing to do with physics, but biotechnology.

Glaser became professor of physics at the University of California, Berkeley, in 1959. However, he grew tired of particle physics, which increasingly involved large teams of investigators using big instruments, and became professor of molecular biology in 1964. His passion and skill at building equipment resulted in several new methods for automating large-scale hunts for pharmaceutically valuable mutants in organisms such as bacteria. He then did something that was unusual at the time. In 1971, with Ron Cape and Peter Farley, he co-founded Cetus, the first US biotechnology company, becoming chairman of its science advisory board. This was where Mullis discovered PCR, and other researchers synthesized interferon, a protein that helps to mediate the immune response. The company merged with Chiron in 1991 and was later purchased by Novartis.

As molecular biology became increasingly about biochemistry, Glaser again considered a career change. Partly because of his love of visual art, and because of problems he had encountered while training workers to recognize bubble-chamber tracks or assess the growth of cultures, he became fascinated by human vision. It was through this new interest that I met Glaser in 1981, and again in 1982 in Cambridge, Massachusetts, during his mini-sabbatical at the Rowland Institute - now part of Harvard University - where Edwin Land, the founder of Polaroid, was studying human perception of colour.

In the early 1980s, Glaser worked on visual psychophysics - a body of quantitative methods to measure perception - and on computational modelling of the visual system. From 1989, his position at Berkeley was professor of physics and neurobiology.

Don and I had stayed in close touch since 1981. I loved our discussions and the observations he made, which were simple, crisp and deep. It is both a privilege and a burden to win a Nobel prize at such a young age. And I always admired the grace with which Don carried his success in science and in industry. Somehow he retained the happy curiosity of a child: the world for him was a garden of wonders. He was able, in an apparently effortless way, to come up with refreshingly counterintuitive observations of things and of people.

Tomaso Poggio is professor in the Department of Brain and Cognitive Sciences at the McGovern Institute and at the Artificial Intelligence Laboratory, Massachusetts Institute of Technology, Cambridge, Massachusetts 02139, USA. e-mail:tp@csail.mit.edu 